\title{
Generalized beams and continua. Dynamics of reticulated structures.
}

\author{
Boutin C. , Hans S. and Chesnais C.
}

\begin{abstract}
This paper deals with the dynamic behaviour of periodic reticulated beams and materials. Through the homogenization method of periodic discrete media the macro-behaviour is derived at the leading order. With a systematic use of scaling, the analysis is performed on the archetypical case of symmetric unbraced framed cells. Such cells can present a high contrast between shear and compression deformability, conversely to "massive" media. This opens the possibility of enriched local kinematics involving phenomena of global rotation, inner deformation or inner resonance, according to studied configuration and frequency range.
\end{abstract}

\section{Introduction}

This paper deals with the macroscopic dynamic behaviour of periodic reticulated structures and materials widely encountered in mechanical engineering. Periodic lattices have been studied through various approaches such as transfer matrix, variational approach [8], finite difference operator, cf. [10]. Asymptotic methods of homogenization [11] initially developed for periodic media, were extended to multiple parameters and scale changes by [5] and adapted to periodic discrete structures by [3], then [9]. Those studies aim at relate the local structure and the global behavior.

The structural morphology of reticulated media makes that the basic cells can present a high contrast between shear and compression deformability (conversely to "massive" media). This opens the possibility of enriched local kinematics involving phenomena of global rotation, inner deformation or inner resonance, according to studied configuration and frequency range. A numerical illustration of these atypical situations is given on Fig. 1 that shows the some unusual macroscopic modes.

Boutin C. , Hans S. and Chesnais C.

DGCB, FRE CNRS 3237, Ecole Nationale des Travaux Publics de l'Etat, Université de Lyon, e-mail: claude.boutin@entpe.fr ; stephane.hans@entpe.fr ; celine.chesnais@entpe.fr 


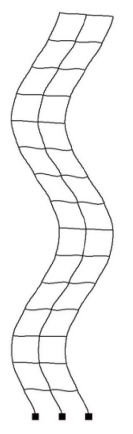

(a)

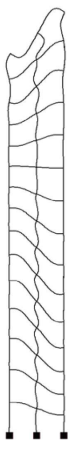

(b)

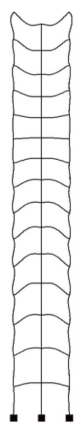

(c)

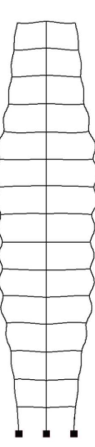

(d)

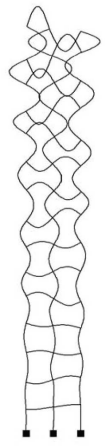

(e)
Fig. 1 Examples of typical and atypical dynamic behaviours of 1D framed structures.

The present study investigates and summarizes those phenomena by a systematic analysis performed on the archetypical case of symmetric unbraced framed cells [1], [7]. Assuming the cell size is small compared to the wavelength, the homogenization of periodic discrete media leads to the macro-behaviour at the leading order.

The paper is organized as follows. Section 2 gives an overview of the method and the assumptions. In section 3, several beam modeling under transverse vibrations are established by varying the properties of the basic frame elements, and the frequency range. Section 4 is devoted to longitudinal vibrations. Section 5 focuses on the analogy between these results and the mechanics of generalized continua.

\section{Discrete homogenization}

The analysis of periodic beam lattices is performed in two steps [12] : first, the discretization of the balance of the structure under harmonic vibrations; second, the homogenization, leading to a continuous model elaborated from the discrete description. An outline of this method is given hereafter.

Discretization of the dynamic balance: Studied structures (Fig.2) are made of plates behaving as Euler-Bernoulli beam in out-of-plane motion, and assembled with rigid connections. The motions of each extremity connected to the same node are identical and define the discrete nodal kinematic variables of the system. The discretization consists in integrating the dynamic balance (in harmonic regime) of the beams, the unknown displacements and rotations at their extremities taken as boundary conditions. Forces applied by an element on its extremities are then expressed as functions of the nodal variables. The balance of each element being satisfied, it remains to express the balance of forces applied to the nodes. Thus, the balance of the whole structure is rigorously reduced to the balance of the nodes. 
Homogenization method: The key assumption of homogenization is that the cell size $\ell$ is small compared to the characteristic size $L$ of the vibrations of the structure. Thus $\varepsilon=\ell / L<<1$. The existence of a macro scale is expressed by means of macroscopic space variable $x$. The physical variables are continuous functions of $x$ coinciding with the discrete variables at any node, e.g. $U_{\varepsilon}\left(x=x_{n}\right)=U($ node $n)$. These quantities, assumed to converge when $\varepsilon$ tends to zero, are expanded in powers of $\varepsilon: U_{\varepsilon}(x)=U^{0}(x)+\varepsilon U^{1}(x)+\varepsilon^{2} U^{2}(x)+\ldots$. Similarly, all unknowns, including the modal frequency, are expanded in powers of $\varepsilon$. As $\ell=\varepsilon L$ is a small increment with respect to $x$, the variations of the variables between neighboring nodes are expressed using Taylor's series; this in turn introduces the macroscopic derivatives.

To account properly for the local physics, the geometrical and mechanical characteristics of the elements are scaled according to the powers of $\varepsilon$. As for the modal frequency, scaling is imposed by the balance of elastic and inertia forces at macro level. This scaling insures that each mechanical effect appears at the same order whatever the $\varepsilon$ value is. Therefore, the same physics is kept when $\varepsilon \rightarrow 0$, i.e. for the homogenized model. Finally, the expansions in $\varepsilon$ power are introduced in the nodal balances. Those relations, valid for any small $\varepsilon$, lead for each $\varepsilon$-order to balance equations whose resolution defines the macroscopic governing equations.

Inner quasi statism and inner dynamics : In general the scale separation requires that, at the modal frequency of the global system, wavelengths of the compression and bending vibrations generated in each local element are much longer than the element length. In that case the nodal forces can be developed in Taylor's series with respect to $\varepsilon$. This situation corresponds to a quasi static state at the local scale. Nevertheless, in higher frequency range, it may occurs that only the compression wavelength is much longer than the length elements while local resonance in bending appears. The homogenization remains possible through the expansions of the compression forces and leads to atypical descriptions with inner dynamics. Above this frequency range, the local resonance in both compression and bending makes impossible the homogenization process.

\section{Studied structures}

We study the vibrations of structures of height $H=N \cdot \ell_{w}$ constituted by a pile of a large number $N$ of identical unbraced frames called cells (Fig.2). The parameters of horizontal elements $(i=f)$ and vertical elements $(i=w)$ are: length $\ell_{i}$; thickness $a_{i}$; section area $A_{i}$; inertia $I_{i}=a_{i}^{3} h / 12$ in direction $e_{3}$; density $\rho_{i}$; elastic modulus $E_{i}$.

The kinematic is characterized at any level $n$ by the motions of the two nodes in the plane $\left(e_{1}, e_{2}\right)$, i.e., the displacements in the two directions and the rotation $\left(u_{1}, u_{2}, \theta\right)$. These six variables can be replaced by (i) three variables associated to the rigid body motion of the level $n$ : the mean transverse displacements, $U(n)$ along $e_{1}$, $V(n)$ along $e_{2}$ and the rotation $\alpha(n)$ (differential vertical nodal motion divided by $\ell_{p}$ ) and (ii) three variables corresponding to its deformation : the mean and differential 


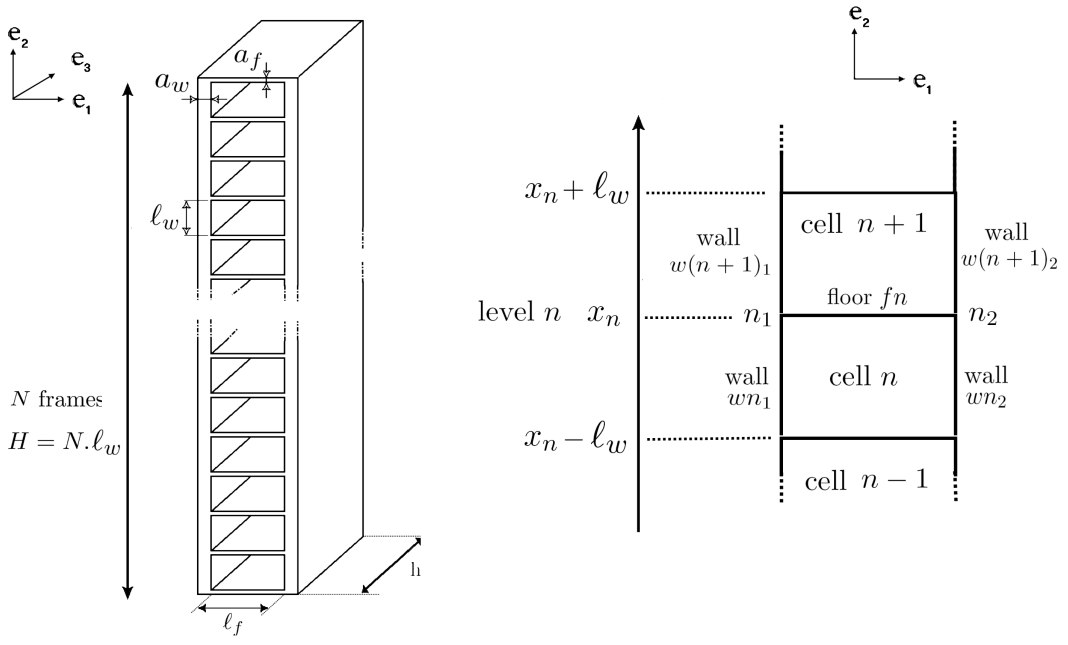

Fig. 2 The class of unbraced framed structures (left) and the basic frame and notations (right).

rotations of the nodes, $\theta(n)$ and $\Phi(n)$, and the transverse dilatation $\Delta(n)$, cf. Fig.3. Because of the longitudinal symmetry the transverse and longitudinal kinematics respectively governed by $(U, \alpha, \theta)$ and $(V, \Phi, \Delta)$ are uncoupled.

A systematic study enables to identify the family dynamic behaviors by evolving gradually the properties of the frame elements and the frequency range.

\section{Transverse vibrations}

The transverse vibrations can be classified in two categories. For the first category, the horizontal elastic force balances the horizontal translation inertia. In this situation, it can be shown that the corresponding range of frequency is such that the cell behaves in the quasi static range. Lower frequencies would lead to a static description of the structure. Conversely, at higher frequencies the horizontal elastic forces cannot balance the translation inertia so that the translation should vanish. Then the vibration results from the balance between the elastic momentum and the rotation inertia. This leads a second unusual category.

\subsection{Low frequency range - Generic beam model}

The possible beam-like behaviours are established by varying the properties of the basic frame elements. The synthesis of the different macroscopic behaviors derived 
Fig. 3 Decoupling of transverse (left) and longitudinal (right) kinematics.

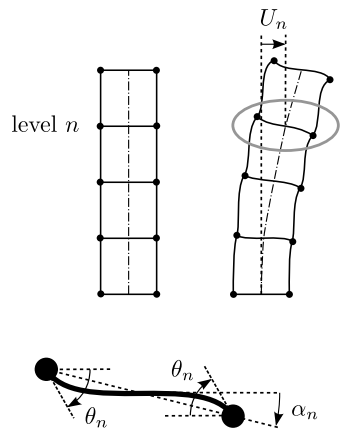

according to the properties of the frame elements shows that only three mechanisms - shear, global bending, inner bending - governs the physics at the macroscale. Each of them is associated to an elastic cell parameter of stiffness : in shear $K$, in global bending $E_{w} I$, and in inner bending $2 E_{w} I_{w}$. Owing to the quasi static local state, these parameters are deduced from the elastic properties of elements in statics.

The method enables to build a generic beam model involving the three kinematic variables $U, \alpha, \theta$ and including the three mechanisms. It is governed by the following sixth order equation :

$$
\frac{2 E_{w} I_{w} E_{w} I}{K} U^{(6)}(x)-\left(2 E_{w} I_{w}+E_{w} I\right) U^{(4)}(x)-\frac{E_{w} I}{K} \Lambda \omega^{2} U^{\prime \prime}(x)+\Lambda \omega^{2} U(x)=0
$$

Eq. (1) generalizes the classical beam dynamics. In addition to the common bending, it includes at the leading order shear and inner bending. Usual descriptions (as Timoshenko, Euler-Bernoulli, Shear) can be recovered by vanishing one or two of these mechanisms. Note that for this category, the rotation inertia of the section do not appear, while the shear may governs the behavior of slender structures.

The dominating effect(s) that drive(s) the effective behavior of a given structure, can be identified through a dimensional analysis performed on the generic beam. Introducing the characteristic size of vibration $L$ the change of variables $\boldsymbol{x}=x / L$ transforms the governing equation (1) into:

$$
C \gamma U^{*(6)}(\mathbf{x})-(1+\gamma) U^{*(4)}(\mathbf{x})-\Omega^{2} U^{*(2)}(\mathbf{x})+\frac{\Omega^{2}}{C} U^{*}(\mathbf{x})=0
$$

where, by construction, the dimensionless terms denoted by a * are $O(1)$ and using for $L$ the actual vibration's size $\tilde{L}=\frac{2 H}{\pi}$ (for a clamped-free beam):

$$
C=\frac{E_{w} I}{K \tilde{L}^{2}} \quad \gamma=\frac{2 E_{w} I_{w}}{E_{w} I}=\frac{2 I_{w}}{I} \quad \Omega^{2}=\frac{\Lambda \omega^{2} \tilde{L}^{2}}{K}
$$

$C$ evaluates the contrast between global bending and shear, and $\gamma$ the contrast between inner and global bending. $C$ and $\gamma$ supply identification criteria of behavior: according to their order of magnitude with respect to $\tilde{\varepsilon}=\ell / \tilde{L}=\frac{\pi}{2 N}$, equation (2) 


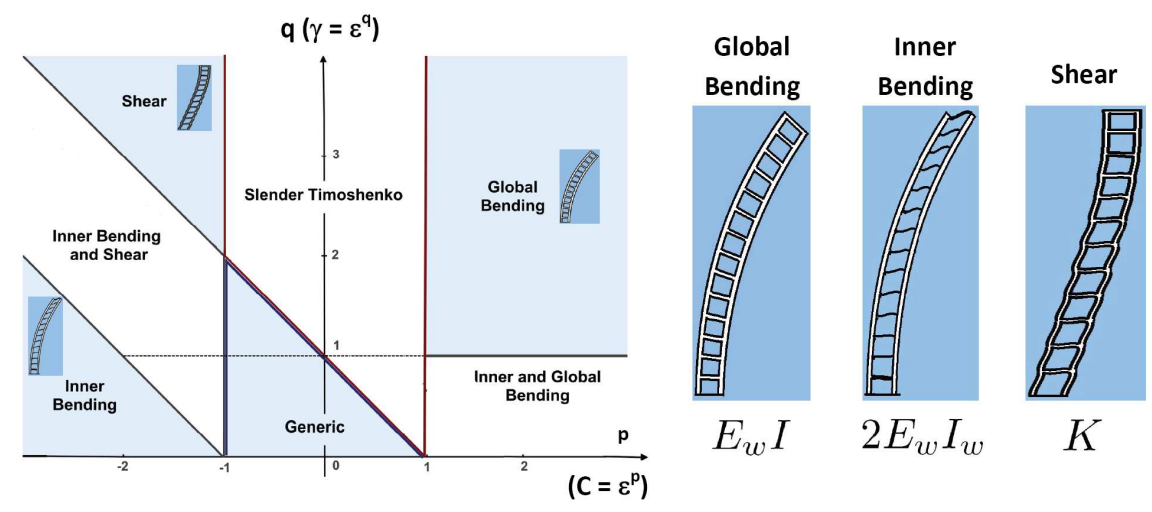

Fig. 4 Left: map of transverse behaviours in function of the dimensionless parameters $C=\tilde{\varepsilon}^{p}$ and $\gamma=\tilde{\varepsilon}^{q}$. Right : the three transverse mechanisms

degenerates in simplified forms. For instance, if $C=O(1)$ and $\gamma=O(\tilde{\varepsilon})$, the terms related to $C \gamma$ and $\gamma$ disappear and the resulting model is:

$$
U^{*(4)}+\Omega^{2} U^{*(2)}-\frac{\Omega^{2}}{C} U^{*}=0
$$

that corresponds to a slender Timoshenko beam, etc... Doing so, seven behaviors are obtained depending on the value of $C, C \gamma$ and $\gamma$ compared to $\tilde{\varepsilon}$ powers. A synthetic representation (Fig. 4) is deduced by mapping the domain of validity of each behavior according to the two parameters $\mathrm{p}$ and q defined by: $C=\tilde{\varepsilon}^{p}$ and $\gamma=\tilde{\varepsilon}^{q}$

\subsection{Higher frequency range - Gyration beam model}

At higher frequencies the translation $U$ must vanish at the leading order (when seeking for a macroscopic description). A new dynamic equilibrium between elastic momentum and rotation inertia leads to the second category of macroscopic transverse vibrations. The corresponding atypical gyration beam model involves only the two kinematic variables $\alpha, \theta$, driven by the mechanisms of opposite tractioncompression of vertical elements (global bending without translation ! see Fig.1-b) and the frame shear. Because of the higher frequency domain, the condition of local quasi statism is not necessarily respected and thus two models are obtained.

Inner quasi-statism : This situation corresponds to bending (then compression) wavelength much longer than the elements length. The governing equation is of the second degree whose parameters are the elastic stiffnesses already determined and the usual rotation inertia $J$. It reads : 


$$
E_{w} I \alpha^{\prime \prime}(x)-K \alpha(x)+J \omega^{2} \alpha(x)=0
$$

It can be interpreted as the balance of the global momentum with a classical inertial source term and an inner elastic source of momentum.

Inner dynamics : In this case the bending wavelength in the element is of the order of their length, meanwhile the compression wavelength remains much larger. This enables to expand the compression forces and to derive a macro behavior. The governing equation of the second degree presents the same global momentum parameters than for local quasi statism but differs fundamentally by the inertia term and the inner elastic source of momentum, both depending on frequency :

$$
E_{w} I \alpha^{\prime \prime}(x)-K(\omega) \alpha(x)+J(\omega) \omega^{2} \alpha(x)=0
$$

The reason of these modifications lies in the non expanded forces associated to the local dynamic bending that strongly depends on the frequency and gives rise to apparent inertia $J(\omega)$ and momentum source. This effect also appears in longitudinal vibrations and is discussed in the next section.

\section{Longitudinal vibrations}

The longitudinal vibrations, described by $(V, \Phi, \Delta)$, present a lesser complexity because the main mechanism is the vertical compression. The difference between the identified models only relies in the possible presence of inner dynamics.

Inner quasi-statism : This case leads to the classical description of beam characterized by the compression modulus $2 E_{w} A_{w}$ and the lineic mass $\Lambda$ :

$$
2 E_{w} A_{w} V^{\prime \prime}(x)+\Lambda \omega^{2} V(x)=0
$$

The domain of validity of this model is derived by expressing that the orders of magnitude of the fundamental frequency of the whole structure (described by eq.6) is much smaller than the one of the elements in bending. This leads to the following lower bound of the number of cells : $N \geq$ slender ratio of the elements. In other words the validity of usual model requires a sufficiently large number of cells.

Inner dynamics : Similarly to gyration modes, the inner dynamics introduces an frequency depending apparent mass, that can be expressed analytically [4] :

$$
\begin{array}{r}
2 E_{w} A_{w} V^{\prime \prime}(x)+\Lambda(\omega) \omega^{2} V(x)=0 \quad ; \quad \Lambda(\omega)=\Lambda_{w}+\Lambda_{f} \psi\left(\frac{\omega}{\omega_{f_{1}}}\right) \\
\psi(\tilde{\omega})=\frac{8 \sin \left(\frac{3 \pi}{4} \sqrt{\tilde{\omega}}\right) \operatorname{sh}\left(\frac{3 \pi}{4} \sqrt{\tilde{\omega}}\right)}{\left.3 \pi \sqrt{\tilde{\omega}}\left(\sin \left(\frac{3 \pi}{4} \sqrt{\tilde{\omega}}\right) \operatorname{ch}\left(\frac{3 \pi}{4} \sqrt{\tilde{\omega}}\right)\right)+\operatorname{sh}\left(\frac{3 \pi}{4} \sqrt{\tilde{\omega}}\right) \cos \left(\frac{3 \pi}{4} \sqrt{\tilde{\omega}}\right)\right)}
\end{array}
$$




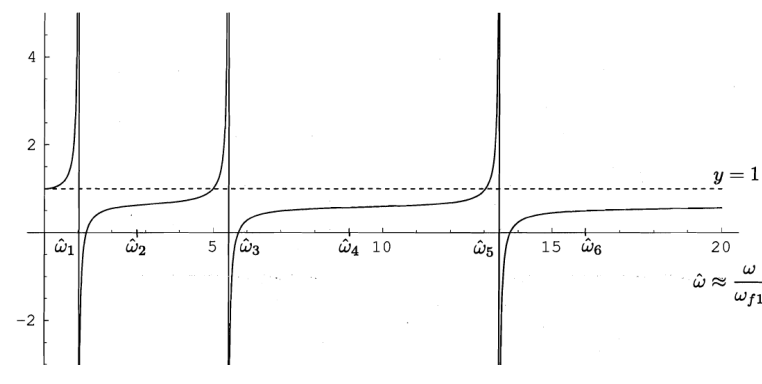

Fig. 5 Effect of the inner resonance on the apparent dimensionless mass : $\left(1+\psi\left(\frac{\omega}{\omega_{f 1}}\right)\right) / 2$

The study of $\Lambda(\omega)$ (cf. Fig. 5), shows that (i) $\Lambda(\omega) \rightarrow \Lambda$ when $\omega \rightarrow 0$, and (ii) $|\Lambda(\omega)| \rightarrow \infty$ when $\omega \rightarrow \omega_{f_{2 k+1}}$, where $\omega_{f_{2 k+1}}$ are the odd eigen modes of horizontal elements in bending. This induces abnormal response in the vicinity of the $\omega_{f_{2 k+1}}$ that results in discrete spectrum of cut-off frequencies. The domain of validity of this model is derived by equalizing the orders of magnitude of the fundamental frequencies of (i) the whole structure and (ii) the elements in bending. This leads to bound of the number of cells by $N \leq$ slender ratio of the elements.

\section{Analogy with micromorphic media}

This section points out analogy between the several beam behaviors and that of micromorphic materials [6]. Conveniently we focus on the first (low frequency) mode of transverse vibration polarised in direction $e_{2}$, propagating in direction $e_{1}$.

\subsection{From $1 D$ to $2 D$ structures}

Consider a cell $\mathscr{F}$ of shear stiffness $K$, inner bending stiffness $E I_{w}$ and global bending stiffness $E I$ corresponding to the intrinsic coefficients $\beta=E I / K l_{w}^{2}$ and $\gamma=I_{w} / I$. From this frame, build a periodic beam $\mathscr{B}=N_{e_{1}} \times \mathscr{F}$, made of $N_{e_{1}}$ frame cells repeated in the direction $e_{1}$, as in Fig.2. The transverse behavior of $\mathscr{B}$ is driven by the two dimensionless parameters and the actual scale ratio :

$$
C=\beta\left(\frac{\pi}{2 N_{e_{1}}}\right)^{2}=\tilde{\varepsilon}^{p} \quad ; \quad \gamma=\tilde{\varepsilon}^{q} \quad ; \quad \tilde{\varepsilon}=\frac{\pi}{2 N_{e_{1}}}
$$

Build the 2D periodic structure $\mathscr{S}$ by $N_{e_{2}}$ lateral repetition in the transverse direction $e_{2}$ of $\mathscr{B}$ that is, $\mathscr{S}=N_{e_{2}} \times \mathscr{B}=N_{e_{1}} \times\left(N_{e_{2}} \times \mathscr{F}\right)$. The properties of the $\mathscr{S}$ 's cell made of $N_{e_{2}} \times \mathscr{F}$ frames can be estimated from those of $\mathscr{F}$ : the stiffnesses in shear is $O\left(N_{e_{2}} K\right)$, in inner bending is $O\left(N_{e_{2}} E I_{w}\right)$, and in global bending is $O\left(N_{e_{2}}^{3} E I\right)$. Thus, the transverse behavior of $\mathscr{S}$ is driven by $C_{S}$ and $\gamma_{S}$ : 


$$
C_{S}=\left(\frac{\pi N_{e_{2}}}{2 N_{e_{1}}}\right)^{2} \beta>C ; \quad \gamma_{S}=\left(\frac{1}{N_{e_{2}}}\right)^{2} \gamma<\gamma \quad \text { then } \quad C_{S} \gamma_{S}=C \gamma=\left(\frac{\pi}{2 N_{e_{1}}}\right)^{2} \beta \gamma
$$

Moreover since $\mathscr{B}$ and $\mathscr{S}$ have the same number $N_{e_{1}}$ of cells : $\tilde{\varepsilon}_{S}=\tilde{\varepsilon}$. Introducing $\left(p_{S}, q_{S}\right)$ such that $C_{S}=\tilde{\varepsilon}^{p_{S}}$ and $\gamma_{S}=\tilde{\varepsilon}^{q_{S}}$ the relations (9) lead to:

$$
p_{S}<p \quad ; \quad q_{S}>q \quad ; \quad p_{S}+q_{S}=p+q
$$

Consequently, according to the previous results (cf. Fig4), the nature of $\mathscr{B}$ and $\mathscr{S}$ behaviour would be the same if $p \leq-1$, but may differ if $p>-1$.

\subsection{Micromorphic media}

Consider now an infinite media made of the cell $\mathscr{F}$ and assume that - in a domain $\mathscr{D}$ restricted to $N_{e_{1}} \times N_{e_{2}} \times \mathscr{F}$ - appropriate boundary conditions impose normally to the direction $e_{1}$ a shear polarised in the direction $e_{2}$. The equivalent behaviour in $\mathscr{D}$ is identified by comparing the power of $\tilde{\varepsilon}=\pi /\left(2 N_{e_{1}}\right)$ with the two parameters :

$$
C_{\mathscr{D}}=\left(\frac{\pi N_{e_{2}}}{2 N_{e_{1}}}\right)^{2} \beta \quad ; \quad \gamma_{\mathscr{D}}=\left(\frac{1}{N_{e_{2}}}\right)^{2} \gamma
$$

Four behaviours may arrise according to the independant kinematic variable(s).

Cauchy elastic continuum behaviour. This will be observed when the kinematic involves the solely translation $U$, which occurs when $C_{\mathscr{D}} \gamma_{\mathscr{D}} \leq \tilde{\varepsilon}$ and $C_{\mathscr{D}} \geq \tilde{\varepsilon}^{-1}$. These conditions require that the $\mathscr{D}$ geometry follows the constraints : in the direction normal to the shear motion $N_{e_{1}} \geq \frac{\pi}{2} \beta \gamma$ i.e. a number of cell higher than the intrinsic critical number $N_{c}=\frac{\pi}{2} \beta \gamma=E I_{w} / K l_{w}^{2}$; in the direction of the shear motion $N_{e_{2}} \geq\left(\frac{2}{\pi} N_{e_{1}}\right)^{3 / 2} / \sqrt{\beta}=M_{2}$, i.e. a weak slenderness aspect ratio of $\mathscr{D}$.

Micromorphic elastic media with inner deformation. This appears when the kinematic involves the translation $U$ and the inner rotation $\theta$, i.e., when $C_{\mathscr{D}} \gamma_{\mathscr{D}} \geq \tilde{\varepsilon}$ and $C_{\mathscr{D}} \geq \tilde{\varepsilon}^{-1}$. In terms of $\mathscr{D}$ geometry this requires that $N_{e_{1}} \leq N_{c}$ and $N_{e_{2}} \geq M_{2}$

Cosserat like behaviour. The kinematic variables are the translation $U$ and the rotation $\alpha$. This appears when $C_{\mathscr{D}} \gamma_{\mathscr{D}} \leq \tilde{\varepsilon}$ and $C_{\mathscr{D}} \leq \tilde{\varepsilon}^{-1}$ i.e. when $N_{e_{1}} \geq N_{c}$ and $N_{e_{2}} \leq M_{2}$. In this case the cell rotation breaks the lateral periodicity, whereas the periodicity in the perpendicular direction is kept. For this reason, such a phenomenon is not be described by the usual 2D homogenization applied the basic $\mathscr{F}$ (that would impose the periodicity in both directions). Nevertheless, the deformation presents a scale separation in a single direction that enables the treatment by a 1D homogenization. In such a domain, the effects of global bending and shear are of same order.

Complex micromorphic media with inner deformation and rotation involving the 
three variables $U, \alpha$ and $\theta$. This situation is obtained when $C_{\mathscr{D}} \gamma_{\mathscr{D}} \geq \tilde{\varepsilon}$ and $C_{\mathscr{D}} \leq \tilde{\varepsilon}^{-1}$ that is for rather restricted domain geometry defined by: $N_{e_{1}} \leq N_{c}$ and $N_{e_{2}} \leq M_{2}$.

To respect the scale separation, the micromorphic behaviours with inner deformation can only be reached if $N_{c}=\frac{\pi}{2} \beta \gamma$ is significantly larger than 1 .

\section{Conclusion}

Reticulated media present a much larger diversity of behavior than the usual "massive" media. In particular the enriched local kinematics introduces modes of different nature (as gyration modes), based on different kind of equilibrium (as inner bending modes). The atypical inner resonance effect is also demonstrated to be possible for those reticulated structure, while they cannot develop in massive beams.

The comparisons of these theoretical results with numerical modelling are satisfactory [7], and dynamics experiments on real regular buildings [2] also demonstrate the reliability of this approach and its interest in engineering domain. Finally these results may be extended to the rheology of reticulated materials such as foam, glass wool, vegetal, bones ... and presents strong analogies with generalized continua.

\section{References}

1. Boutin, C., Hans, S., 2003 "Homogenisation of periodic discrete medium: Application to Dynamics of framed structures", Computers and Geotechnics, 30(4), pp303-320.

2. Boutin, C., Hans, S., Ibraim, E., Roussillon, P., 2005, "In situ experiments and seismic analysis of existing buildings - Part II", Earthquake Engineering and Structural Dynamics, 34, pp1531-1546.

3. Caillerie, D., Trompette, P., Verna, P.,1989," Homogenisation of periodic trusses", Congres IASS, Madrid, pp 7139-7180.

4. Chesnais C., Hans S., Boutin C., 2007, "Wave propagation and diffraction in discrete structures - Effect of anisotropy and internal resonance" ICIAM 16-20 july Zurich

5. Cioranescu, D., Saint Jean Paulin, J.,, 1999. Homogenization of reticulated structures, Applied Mathematical Sciences 136, Springer.

6. Eringen, A.C.,1968, "Mechanics of micromorphic continua", IUTAM Symposium on the Generalized Cosserat Continuum and the Continuum Theory of Dislocations with Applications, Springer-Verlag (Berlin), pp18-35.

7. Hans, S., Boutin, C., 2008, "Dynamics of discrete framed structures: a unified homogenized description", Journal of Mechanics of Materials and Structures, 3(9), pp1709-1739.

8. Kerr, A.D., Accorsi, M.L., 1985,"Generalization of the equations for frame-type structures a variational approach", Acta Mechanica, 56(1- 2), pp55-73.

9. Moreau, G. and Caillerie, D., 1998, "Continuum modeling of lattice structures in large displacement applications to buckling analysis", Computer and Structures, 68(1-3), pp181-189.

10. Noor, A.K.,1988, "Continuum modeling for repetitive lattice structures", Applied Mechanics Reviews, 41(7), pp285-296.

11. Sanchez-Palencia E.,1980, Non-homogeneous media and vibration theory, Lecture note in physics - Springer-Verlag (Berlin), 127.

12. Tollenaere, H., Caillerie, D., 1998, "Continuum modeling of lattice structures by homogenization", Advances in Engineering Software, 29(7-9), pp699-705. 